University of Wollongong

Research Online

Faculty of Social Sciences - Papers (Archive) Faculty of Arts, Social Sciences \& Humanities

2012

A randomized controlled trial of the efficacy and cost-effectiveness of a brief intensified cognitive behavioral therapy and/or pharmacotherapy for mood and anxiety disorders: Design and methods

\author{
Denise Meuldijk \\ University of Wollongong, meuldijk@uow.edu.au \\ Ingrid V. Carlier \\ Leiden University \\ Irene M. Van Vliet \\ Leiden University \\ M E. Van Den Akker-Van Marle \\ Leiden University \\ Frans G. Zitman \\ Leiden University
}

Follow this and additional works at: https://ro.uow.edu.au/sspapers

Part of the Education Commons, and the Social and Behavioral Sciences Commons

Research Online is the open access institutional repository for the University of Wollongong. For further information contact the UOW Library: research-pubs@uow.edu.au 


\title{
A randomized controlled trial of the efficacy and cost-effectiveness of a brief intensified cognitive behavioral therapy and/or pharmacotherapy for mood and anxiety disorders: Design and methods
}

\begin{abstract}
Background: Anxiety and mood disorders involve a high disease burden and are associated with high economic costs. A stepped-care approach intervention and abbreviated diagnostic method are assumed to increase effectiveness and efficiency of the mental healthcare and are expected to reduce economic costs. Methods: Presented are the rationale, design, and methods of a two-armed randomized controlled trial comparing 'treatment as usual' (TAU) with a brief intensified cognitive behavioral therapy (CBT) and/ or pharmacotherapy. Eligible participants $(\mathrm{N}=500)$ of five Dutch outpatient Mental Healthcare Centers are randomly assigned to either TAU or to the experimental condition (brief CBT and/or pharmacotherapy). Data on patients' progress and clinical effectiveness of treatment are assessed at baseline, posttreatment (3. months after baseline), and at 6 and 12. months post-treatment by Routine Outcome Monitoring (ROM). Cost analysis is performed on the obtained data. Discussion: Since few studies have investigated both the clinical and cost effectiveness of a stepped-care approach intervention and a shortened diagnostic ROM method in both anxiety and/or mood disorders within secondary mental health care, the results of this study might contribute to the improvement of (cost)-effective treatment options and diagnostic methods for these disorders.
\end{abstract}

\section{Keywords}

controlled, trial, efficacy, cost, effectiveness, brief, randomized, intensified, mood, cognitive, behavioral, therapy, pharmacotherapy, methods, design, disorders, anxiety

\section{Disciplines \\ Education | Social and Behavioral Sciences}

\section{Publication Details}

Meuldijk, D., Carlier, I. V. E., van Vliet, I. M., van den Akker-Marle, M. E. \& Zitman, F. G. (2012). A randomized controlled trial of the efficacy and cost-effectiveness of a brief intensified cognitive behavioral therapy and/or pharmacotherapy for mood and anxiety disorders: Design and methods. Contemporary Clinical Trials, 33 (5), 983-992. 


\title{
A randomized controlled trial of the efficacy and cost- effectiveness of a brief intensified cognitive behavioral therapy and/or pharmacotherapy for mood and anxiety disorders: design and methods
}

\author{
Denise Meuldijk $^{\mathrm{a}^{*}}, \mathrm{MSc}$, Ingrid V.E. Carlier ${ }^{\mathrm{a}}$, PsyD, PhD, Irene M. van Vliet ${ }^{\mathrm{a}}$, \\ MD, PhD, M. Elske van den Akker-Marle ${ }^{b}$, PhD, Frans G. Zitman ${ }^{a}$, MD, PhD \\ a. Department of Psychiatry, Leiden University Medical Center, Leiden, the \\ Netherlands \\ b. Department of Health Economics/Operations Research, Leiden \\ University Medical Center, Leiden, the Netherlands
}

\begin{abstract}
*Corresponding author: D. Meuldijk, MSc, Leiden University Medical Center, Department of Psychiatry, P.O. Box 9600, 2300 RC, Leiden, the Netherlands. Tel: +31-(0)71-5263785, Fax: +31-(0)71-5248156, e-mail: d.meuldijk@lumc.nl
\end{abstract}

Conflict of interest: No disclosures to report.

Funding: See details in the Acknowledgments section

Trial registration: Netherlands Trial Register NTR2590 


\section{Abstract}

Background: Anxiety and mood disorders involve a high disease burden and are associated with high economic costs. A stepped-care approach intervention and abbreviated diagnostic method are assumed to increase effectiveness and efficiency of the mental healthcare and are expected to reduce economic costs.

Methods: Presented are the rationale, design, and methods of a two-armed randomized controlled trial comparing 'treatment as usual' (TAU) with a brief intensified cognitive behavioral therapy (CBT) and/or pharmacotherapy. Eligible participants ( $N=500)$ of five Dutch outpatient Mental Healthcare Centers are randomly assigned to either TAU or to the experimental condition (brief CBT and/or pharmacotherapy). Data on patients' progress and clinical effectiveness of treatment are assessed at baseline, post-treatment ( 3 months after baseline), and at 6 and 12 months post-treatment by Routine Outcome Monitoring (ROM). Cost analysis is performed on the obtained data.

Discussion: Since few studies have investigated both the clinical and cost effectiveness of a stepped-care approach intervention and a shortened diagnostic ROM method in both anxiety and/or mood disorders within secondary mental health care, the results of this study might contribute to the improvement of (cost)-effective treatment options and diagnostic methods for these disorders.

\section{Keywords}

Cognitive behavioral therapy; Brief therapy; Cost-effectiveness; Anxiety and mood disorders; Routine Outcome Monitoring 


\section{Introduction}

Anxiety and mood disorders belong to the most common mental disorders. Several epidemiological surveys found anxiety and mood disorders to be the most prevalent class of mental disorders in the general population $[1,2]$. The World Health Organization surveys estimated their global lifetime prevalence to be $14.3 \%$ and $10.6 \%$, respectively $[1,3]$. These disorders frequently start early in life and tend to have a chronic or relapsing course [4]; moreover, their presence contributes to a high disease burden for both the patient and their family [3]. They also have a substantial impact on daily functioning at home/work and on quality of life [5-8], comparable to the impact and effects of major chronic illnesses $[5,9,10]$. Consequently, the economic costs of these disorders for healthcare systems and society are high [11]. It is estimated that in 2010 the direct and indirect costs of anxiety and mood disorders in Europe were $74.4+113.4$ billion euro, respectively [12]. Earlier studies in the USA and UK reported even higher estimates [13].

In the last decades, evidence-based treatments for anxiety and mood disorders have become available, i.e. cognitive behavioral therapy (CBT) and interpersonal therapy specifically developed for these disorders, as well as pharmacotherapy (mainly antidepressants) [14,15]. As important differences in effectiveness between the treatments are absent [13] guidelines were developed advocating a stepped-care model $[16,17]$. Moreover, to enhance effectiveness, for each of these treatments protocols and guidelines became available based on 
those used in randomized controlled trials (RCT) [4,18-20]. Recently, we added Routine Outcome Monitoring (ROM) to the stepped-care approach to help the diagnostic process and treatment evaluation $[19,20]$.

It is clear that guidelines, protocols and ROM have the potential to improve treatment efficacy. However, these improvements may not yet be fully realized as adherence to the guidelines and protocols remains questionable [17,21-23], even when ROM is added $[19,20]$. This implies that treatments last longer, consist of too many sessions and, thus, unnecessarily prolong suffering and increase related costs $[17,21,22]$. Moreover, the current economic situation offers a strong incentive to make treatments as cost-effective as possible. This applies not only to the treatments as such, but also to ROM; from an economic point of view ROM should be as concise as possible.

As most patients are treated in the first phase of the stepped-care model, it is in this phase that cost reduction is most profitable. Brief therapy is suggested to be suitable as a first step in a stepped-care model $[22,24]$.

This paper describes an RCT designed to evaluate the effects and costs of a shortened first treatment in the stepped-care protocol for anxiety and mood disorders in secondary care, an area where there is a paucity of research.

It consists of time-limited (brief) CBT and/or medication treatment using a protocol following the multidisciplinary guidelines, but confined to a 7-week period and a maximum of 7 sessions. Also examined is the feasibility of a shorter, less labor-intensive ROM. In addition, the cost-effectiveness of the treatment and the adapted ROM are compared to 'treatment as usual' (TAU). 


\section{Design and Method}

\subsection{Study goals}

Primary aim of this study is to evaluate the efficacy and effectiveness of a newly developed time-limited (brief) therapy intervention compared with TAU. Secondary aim is to evaluate the cost-effectiveness of the experimental intervention as compared with TAU.

Additionally, the feasibility of a shortened, less work intensive and timeconsuming ROM is evaluated. Patient and therapist satisfaction with the new intervention is also explored.

\subsection{Study design}

The study is a pragmatic, two-armed RCT using a parallel group design. Five Dutch mental health clinics are projected to enroll a total of 500 participants over an 18-month period of active recruitment. Eligible participants who provide informed consent are randomly assigned to one of two groups: the control group (TAU), or the experimental group. Patients in both groups are assessed by ROM at baseline and after 3 months (post-treatment). Follow-up assessments are conducted in all patients at 6 and 12 months post-treatment.

Primary and secondary outcomes are assessed by ROM. Primary outcomes are the scores on the Web Screening Questionnaire (WSQ) and Brief 
Symptom Inventory (BSI). Secondary outcomes are the scores on the other instruments assessed by ROM (see section 2.8.1 and Table 1).

The design and methodology of this study allows to assess analysis of equivalency (non-inferiority), since we do not expect to find the introduced intervention to be superior to TAU.

\subsubsection{Control Group (TAU)}

Individuals assigned to the control group receive standard psychiatric treatment called; Treatment As Usual (TAU). TAU varies across centers depending on the current activities at the participating MHCs. In MHCs, TAU is not strictly formalized; a multidisciplinary team is free to assign a therapy from a wide range of evidence-based therapeutic options (including: pharmacotherapy and psychological treatment, psychosocial interventions, contact with a psychiatric test nurse) according to the stepped-care approach. The treatment decision is based on professional experience, taking into account the specific problems and characteristics of the individual patient. The number of sessions depends on the therapy that is offered and can be weekly or (almost always) at a lower frequency of sessions, and are not confined to a maximum of sessions.

\subsubsection{Experimental Group}

The experimental group receives a brief, intensified cognitive CBT and/or pharmacotherapy confined to a fixed time period (7 weeks) and a limited number of weekly sessions (maximum 7 sessions within 7 weeks). The offered CBT and 
pharmacotherapy are described in more detail in section 2.9. An intake and outtake session are also involved when in the experimental group (described in section 2.8.2).

\subsection{Study setting}

The study is conducted at five outpatient mental health clinics from the Dutch Regional Mental Health Provider (RMHP) Rivierduinen (RD).

$\mathrm{RD}$ provides secondary mental health care for an area with over one million inhabitants. In the Netherlands access to mental health care is easy and is not limited by insurance or the financial means of the individual patient. Health insurance is compulsory for all citizens and regulated by the government $[25,26]$. The Dutch mental healthcare system is organized in a stepped-care manner and uses evidence-based treatment guidelines. According to a stepped-care approach a brief but intensive first step is offered and patients who are

insufficiently helped by the initial intervention are allowed to 'step up' to subsequent treatment [16]. The therapeutic principles within the treatment protocols of the intervention are referred to as a 'first step' of a stepped-care approach.

\subsection{Participants}

Eligible participants are males and females aged 18 to 65 years, currently diagnosed with an anxiety and/or depressive disorder as main diagnosis. Patients with current psychotic or bipolar traits, homicidal or suicidal risk or 
severe social dysfunction, as diagnosed by their general practitioner (GP), psychiatrist, or as assessed in a diagnostic interview, are excluded. All eligible subjects need to have adequate understanding of the Dutch language.

Patients with the following DSM IV [27] diagnoses are therefore included: minor or major depressive disorder (single episode or recurrent), depressive disorder NAO, dysthemia, panic disorder (with or without agoraphobia), panic disorder NAO, social phobia, simple phobia, generalized anxiety disorder, obsessive compulsive disorder, posttraumatic stress disorder (type I or single trauma), adjustment disorder (with anxiety and/or depressive mood).Co-morbidity associated with other psychiatric diagnoses (with the exception of psychotic or bipolar disorder) is allowed in order to establish a clinically relevant, broadly representative sample.

\subsection{Sample size}

The sample size was calculated using the method of Cohen [28] and based on review of the available literature of earlier comparable studies. We aimed at detecting an equivalence with an acceptable difference of $5 \%$ on the primary outcome measures WSQ and BSI (see section 2.8.1 and Table 1) and a 15\% maximal difference in outcome scores between TAU and the intervention under the usual assumptions of an $\alpha=0.05$ and power of $80 \%$. This results in an intended total sample size of 500 participants. 


\subsection{Recruitment, screening and enrolment procedures}

All patients referred by their GP to one of the participating MHCs for the treatment of anxiety and/or mood disorders are, initially, eligible to participate in the study.

We adopted the following steps in recruitment: first, all referred patients are globally screened by an experienced psychiatrist for the presence of depression and/or anxiety disorders as current, main problem. This global screening is based on written information provided by the GP containing an interpretation of the patient's current health status and referral for further mental health care; this step does not require face-to-face contact with the patient. Subsequently, the potentially eligible patients are invited for a first ROM assessment. Prior to this first ROM assessment, the psychiatric research nurse conducting the ROM assessment invites the patients to participate in the study. Those who agree to participate are asked to provide written informed consent.

When informed consent is given, the baseline ROM assessment $\left(T_{1}\right)$ according to the study design is conducted. After completion of this assessment, participants' randomization by the research team takes place (see section 2.7). Depending on the randomized treatment condition, final eligibility is assessed during the subsequent intake session by means of the inclusion and exclusion criteria of the study.

Patient enrollment began March 1, 2010 and will end December 31, 2011. Follow-up assessment is ongoing and is projected to continue until December 31 , 2012. 


\subsection{Randomization and blinding}

After successful screening, provision of written informed consent and completion of the baseline measurement (T1); (see section 2.6), all eligible participants in this RCT are randomly assigned to one of two groups: the experimental group or the control group (TAU). Random allocation was generated by using a variable blocked design developed by an independent researcher from the Department of Medical Statistics \& Biolnformatics, LUMC and derived by computer.

Randomization takes place on the individual level by clinical center $(n=5)$ and gender. This procedure is used to increase the likelihood that the distribution between groups is balanced on the two potentially important confounding variables and to conceal random allocation sequence.

Participants and clinicians are informed about the outcome of the randomization; the psychiatric test nurses (assessors) involved in the ROM assessment in the study, are kept blinded to the randomization condition throughout the entire study.

Randomization and the subsequent assignment of participants to the intervention will be performed by the researcher (D.M.), whom is not an assessor.

\subsection{Assessment}

For both treatment conditions assessment information is obtained in two-fold, as shown in Figure 1.

\section{$>$ Insert Figure 1 here.}


First, patients participating in this study are assessed by ROM (see 2.8.1.) at four time intervals: 1) $T_{1}$ at baseline (start of study), 2) $T_{2}$ immediately post treatment ( 3 months after baseline measurement), 3) $T_{3} 6$ months post-treatment (first follow-up measurement), and 4) $T_{4} 12$ months post-treatment (second follow-up measurement). The second assessment method is provided by an intake and outtake evaluation (see also 2.8.2).

The timetable of assessments is shown in Figure 1.

\subsubsection{Routine Outcome Measures and feedback}

This study is conducted on data collected using ROM [19]. ROM is a monitoring system for patient care, implemented in 2002 in the outpatient clinics of RD and the Department of Psychiatry of the LUMC. All outpatients referred to these clinics by their GP for treatment of a mood, anxiety and/or somatoform disorder, are assessed by ROM.

ROM periodically measures the presence and severity of psychiatric symptoms in patients and thereby monitors patients' progress/changes during treatment by conducting an extensive battery of psychometric instruments. An overview of the instruments used in ROM is available at http://www.lumc.nl/psychiatry/ROM-instruments.

These instruments are routinely assessed at baseline and during treatment at several time points $[19,29]$. The baseline assessment also comprises a standardized diagnostic interview (Dutch version of the Mini- 
International Neuropsychiatric Interview Plus, version 5.0.0) [30,31], the collection of socio-demographic and socio-economic data, and the administration of general measures of health and disease-specific severity scales.

Instruments are both self-report and interviewer based. All interviewerbased instruments are carried out by a psychiatric research nurse and the selfreport questionnaires are completed using a touch-screen computer. A webbased software QuestManager (www.psyquestmanager.nl) was developed to assist the ROM method and is also used in the current study.

Data collected by ROM are provided to the clinician and patient as written feedback (a brief report) by the psychiatric research nurse. This written feedback consists of an overview of the main measurement results. Furthermore, a summary of the diagnostic interview and a summary of the main questionnaires is given (one or two pages). The clinician shares and discusses these results with the patient. The assessment outcomes are used to support decision-making for the future course of the treatment $[19,29]$.

For the present study, all eligible patients are routinely assessed by ROM. Table 1 lists the instruments used to assess the disorders of interest for the current study. To further test the hypotheses of the present study, five additional questionnaires are added to the regular ROM and are indicated (in bold print) in Table 1.

\section{Insert Table 1 here.}


Similar to the regular ROM, the baseline assessment comprises the standardized diagnostic interview, MINI-Plus 5.0.0, and the collection of sociodemographic and socio-economic data.

For the present study, the written ROM feedback depends on the randomized treatment condition. Clinicians in the experimental group are provided with minimal information about the results of the questionnaires, i.e. an overview of the results of the patient's performance on the two primary outcome measures: the Web Screening Questionnaire (WSQ) [32] and the Brief Symptom Inventory (BSI) [33]. The summary of the diagnostic interview and information about the other measures is left out. Clinicians in the TAU group are provided with the regular, extensive, feedback about all measurement results.

\subsubsection{Intake and outtake}

In addition, to examine the progress of patients' wellbeing and the effectiveness of the treatment, an intake and outtake session is included in the study design (Figure 1). Both intake and outtake session are semi- structured and conducted by the same experienced psychiatrist (see 2.8.5), during one 45 minute (approximately) session.

The intake takes place before the start of the treatment, after the ROM baseline measurement. A semi-structured clinical interview, especially designed for this study, is administered. Compared to a regular intake, this intake session is protocolized, shorter and more structured. Moreover, the intake aims to ensure that the patient is eligible to participate in this study by means of the in- and 
exclusion criteria (section 2.4). Personal data, including demographics and the current clinical picture, are also obtained during this intake session.

The outtake evaluation takes place within (maximally) 2 weeks posttreatment (Figure 1) and is part of a stepped-care approach. The aim of this semi- structured outtake session is to evaluate if patients are sufficiently helped by the offered initial intervention or 'stepping up' to subsequent (additional) treatment is necessary. The 'stepping-up' is according to clinical experience and the local and national guidelines as handled by the involved MHC. During the outtake the progress of the patient's symptoms and his/her current clinical status will be assessed. Furthermore, it evaluates the patient's wellbeing and an 'end diagnosis' is formulated. A possible subsequent treatment plan can be discussed by patient and clinician.

When the outtake session demonstrates that the achieved treatment effect is insufficient, the patient is offered to 'step up' to additional treatment according to the treatment guidelines in the same $\mathrm{MHC}$, or elsewhere.

\subsection{Treatment}

For the current study the following treatment protocols are formulated by the research team:

1. Pharmacotherapy protocol for mood and/or anxiety disorders (maximum 4 sessions within 7 weeks)

2. Brief Cognitive Behavioral Therapy protocol for depression (minimal 5 , maximum 7 weekly sessions) 
3. Brief Cognitive Behavioral Therapy protocol for anxiety (minimal 5, maximum 7 weekly sessions)

4. Eye Movement Desensitization and Reprocessing (EMDR) therapy protocol for post-traumatic stress disorder (PTSD) (maximum 6 sessions within 7 weeks)

The treatment decision was made by the patient and therapist, based on the professional experience of the therapist and taking into account the specific disorder and characteristics of the individual patient, thereby acknowledging the patient's preferences as is good practice and according to the common accepted principles of shared-decision making $[34,35]$.

The treatment protocols are based on the existing treatment guidelines described in the (multidisciplinary) guidelines in Dutch mental healthcare $[4,36,37]$ and on acknowledged evidence-based literature [4,38-41]. All treatment protocols are evaluated during an outtake session (see section 2.8.2.).

Delivering treatment by combining the described protocols is also possible.

\subsubsection{Pharmacotherapy for mood and/or anxiety disorders}

In the current study the pharmacotherapy protocol for mood disorders and/or anxiety is characterized by a quick onset and aims to reach an optimal clinical effect of the used medication, involving rapid stepping up to the most optimal dose and minimizing the side-effects for patients as much as possible. Patients are treated with a selective serotonin reuptake inhibitor (SSRI) in 4 sessions 
within a 7-week period. Medication use is evaluated after this fixed period and continued when necessary. The protocol provides a scenario for patients when not using an SSRI (SSRI 1 condition) or, on the other hand, a scenario for patients with a history of (sufficient and adequate) SSRI use and now starting with a new different SSRI (SSRI 2 condition). When patients are currently using an SSRI (as prescribed by their GP), medication is continued and when necessary adapted according to the pharmacotherapy protocol.

\subsubsection{Brief CBT for depression}

The brief CBT for depression protocol is focusing on decreasing the depressive mood of the client and maintain this improvement and based on the cognitive behavioral therapy manuals/protocols for depression [38,42].

For the current study this protocol is confined to a maximum of 7 weekly sessions (minimal 5 sessions) within a 7 week time-period. The first 3 sessions of this brief CBT protocol are dedicated to activation-enhancement and training of social skills. The last 4 sessions emphasizes tracing and altering irrational cognitions by challenging them $[38,42]$. Each treatment session consists of a 45 -min face-toface contact and is characterized by a quick onset (no waiting list).

\subsubsection{Brief CBT for anxiety}

The brief CBT protocol for anxiety disorder is also characterized by a quick onset (no waiting list) and a maximum number of sessions (minimal 5, maximum 7 
weekly sessions within a 7-week period). Each treatment session consists of a 45-min face-to-face contact.

The main focus of the brief CBT for anxiety protocol is on the core CBT techniques for the treatment of anxiety disorders: anxiety/tension- reducing techniques, cognitive techniques and exposure techniques (used when adequate) [40]. If dysfunctional worrying is interfering, anti-worrying techniques are offered.

\subsubsection{Eye Movement Desensitization and Reprocessing Therapy}

The EMDR therapy for the PTSD protocol is characterized by a quick onset (no waiting list) and a limited number of sessions (a maximum of 6 sessions within 7 weeks). Furthermore, all EMDR sessions comprise a 45-min face-to-face contact. The EMDR protocol of this study is based on the Dutch Manual EMDR [43] and approved principles described in the literature [39], or the treatment of PTSD for patients suffering from a Type I trauma or single trauma.

\subsection{Therapist selection, training and supervision}

The treatment protocols are performed by experienced clinicians: psychiatrists and psychologists working at the participating MHCs.

Clinicians working at RD provide treatment in accordance with the multidisciplinary guidelines of the National Steering Committee describing evidenced-based treatments for mood and anxiety disorders. The participating clinicians are all professionally educated and trained in CBT. Years of experience 
ranged between 1- 7 years. They were especially trained to work with the brief treatment protocols within this study.

The participating clinicians were initially instructed by the research team. Two hours of instructions were provided for participating clinicians on two consecutive days. On the first day, a 2-hour instruction was provided for clinicians involved in the CBT condition. During this instruction, the EMDR protocol was also introduced. On the subsequent day, a 2-hour instruction was provided for clinicians involved in providing the pharmacotherapy within this study.

During these instruction meetings, consensus was achieved on a number of core elements of the treatments. Furthermore, the content of future supervision session for all involved clinicians was discussed.

Clinicians in the TAU group received no specific training from the research team. They provide the usual treatment to the patients; globally following the available multidisciplinary treatment guidelines, since adherence is questionable.

Furthermore, six certified psychiatric test nurses were trained by the research team to assess the ROM measurements according to the guidelines of the study. The research nurses are all trained and certified to assess ROM measurements.

Instruction was given on the administration and reporting of the ROM measurements designed for this study. The psychiatric test nurses were informed about the logistics of the study and how to apply the blinding method used in the present study. 
Once every 3 months supervision sessions were organized by the research team for the clinicians and the psychiatric test nurses. Interview techniques, how to avoid protocol violations and other challenges were discussed and evaluated.

In addition, unrestricted support is provided to all study clinicians and psychiatric test nurses via email and through visits made by the research team.

\subsection{Fidelity monitoring}

All treatment sessions within the brief CBT protocols will be audio-taped, using a digital voice recorder to ensure treatment protocol fidelity. The taped treatment sessions will be randomly checked and scored on protocol consistency and reliability by the research team. Furthermore, therapist adherence and satisfaction will be monitored using an evaluation questionnaire. This evaluation questionnaire furthermore monitors the delivery and compliance of the different treatment protocols since it questions how many sessions were involved and if treatment was successfully delivered. This evaluation questionnaire, and the list of the criteria for protocol consistency and reliability, can be obtained from the corresponding author.

\subsection{Statistical analyses}

Descriptive statistics will be used to describe the characteristics of the two groups, and the outcome variables, at the four measurement points. To evaluate potential group differences at baseline, post-treatment and at the 6 and 12-month 
follow-up measurements, repeated measures analysis will be conducted to analyze the short and long-term effects between the experimental and control group.

All analyses will be conducted according to the intention-to-treat (ITT) approach. Additionally, analysis per protocol will be conducted.

Chi-square analyses and t-tests for independent samples will be used for data measured on one occasion (e.g. patient satisfaction, therapist satisfaction, baseline demographic features) to detect possible differences between the two groups. Differences are considered statistically significant at $p<0.05$.

Missing values will be imputed with regression imputation techniques. Generalized Estimating Equation (GEE) analyses will additionally be performed on the dataset with missing data and when missing values are imputed. Analyses adjusting for cluster effects will be performed when analyzing the data.

All analysis will be done using SPSS (version 17, Windows).

\subsubsection{Health economics analysis}

Cost-effectiveness will be also calculated. An economic evaluation based on the TIC-P questionnaire (see Table 1) examines the costs and other aspects of the study protocol. Direct costs per patient in the experimental condition versus the costs per patient in the control condition will be compared. Costs are validated by reference; if these references are not available, costs will be estimated by costs research. 
Cost or product losses are verified by the 'friction cost method' [44].The friction cost method estimates the indirect costs of disease, which explicitly considers economic circumstances that limit production loss due to disease. According to this method, these indirect costs mainly occur during the time it takes to replace a worker, i.e. the friction period [45].

\subsection{Approvals and data/safety monitoring}

The study protocol was approved by the Medical Ethical Committee (MEC) of the Leiden University Medical Center (LUMC). After full verbal and written information about the study, written informed consent was obtained from all participants at the start of baseline assessment. To safeguard the anonymity of the patients and to ensure proper handling of the data, processing of all data is in accordance with a comprehensive protocol: the Psychiatric University Network REgistration Leiden (PAREL-regulations). The MEC of the LUMC approved the regulations of this protocol and agreed with this policy [19]. Confidentiality of data is maintained by using a unique research identification (ID) number for each participant, which enables to identify individuals without using names. Only a limited number of persons (researcher) have access to the record that links the ID number to identifiable information. 


\section{Current status and demographics of sample}

Data will be collected until at least December 31, 2012. At the time of completion of this paper, the inclusion period of the study is still ongoing. A total of 161 patients have completed the baseline measurement. Their preliminary sociodemographic and clinical characteristics are presented in Table 2.

\section{$>$ Insert Table 2 here.}

These 161 participants are evenly distributed across both groups; 79 in the TAU group and 82 in the experimental group. Demographic data (educational and employment status, and ethnic background) are missing for 4 patients. Moreover, for 12 patients the MINI-Plus diagnostic interview did not lead to a DMS-IV classification in ROM; therefore, only a clinical diagnosis is available for these patients.

At baseline there were no significant differences in demographic data between the two groups. The mean age of the patients is 37.2 (SD 11.5; range 18-65) years, and there is a similar distribution for gender (61.5\% female and $38.5 \%$ male) in both groups.

Furthermore, the participants comprise a relatively homogeneous group with common mental disorders, i.e. mainly mood and anxiety disorders. The majority of the included patients have more than one clinical diagnosis.

At baseline, of all patients $45.3 \%$ had an anxiety disorder only, and $37.9 \%$ were diagnosed with depression only. In total, $15.5 \%$ of the patients were 
diagnosed with both an anxiety and mood disorder. This distribution was similar in both treatment arms (Table 2).

\section{Discussion}

Evidence-based clinical guidelines advocating a stepped-care approach are available in mental healthcare and have demonstrated success in the treatment of mood and anxiety disorders [46]. Progress within these steppedcare approaches is carefully monitored and patients are able to 'step up' when no subsequent improvement occurs [16].

However, initiation of and adherence to these recommended and effective treatments within these guidelines is usually poor [18].The optimal content and organization of how to provide this stepped care is unclear, and implementation and acceptability of stepped care as a method of delivering psychological/ psychiatric services has not yet been adopted [21,22]. Little information is available about how stepped care should be effectively implemented [22] and only a few randomized trials present convincing evidence and evaluations of both the cost and clinical effectiveness of this stepped-care model. Most studies investigated either the cost effectiveness $[47,48]$ or the clinical effectiveness [4952] of stepped care. When simultaneously investigated, the studies examined the effectiveness of a stepped-care model for patients with either a mood disorder [53] or an anxiety disorder [54], and only in primary care.

The present study aims to examine the clinical effectiveness and cost effectiveness of an innovative stepped-care intervention for patients (aged 18-65 
years) with anxiety and/or mood disorders in secondary care. To carefully monitor the patient's progress, the ROM method is added.

To our knowledge this is the first long-term study to simultaneously analyze the clinical and cost effectiveness of a stepped-care approach in the treatment of both mood and anxiety disorders in a secondary care setting. The ROM method, monitoring patients' progress at fixed time intervals with a followup period of 1 year, allows to establish the long-term efficiency expectations of treatment success and effects on health status and economic costs. Moreover, for the current study, ROM is shortened and evaluated in terms of feasibility. Since the baseline characteristics of the participants in both the experimental and the control group show no significant differences, the study outcomes are expected to be highly generalizable.

The findings of the present study will have potential implications for provision of the most convenient form of mental healthcare in the Netherlands. The offered time-limited, brief intervention could provide valuable information to help the development of an optimal treatment protocol for patients with anxiety and/or mood disorders. The limited number of sessions reduces the average amount of therapist input per patient and, moreover, is an adequate response to patient preferences for brief psychological interventions [55]. Since the intervention has not only fewer sessions but also an earlier start after intake, it is expected to reduce waiting lists; this is a common problem in the provision of mental healthcare. 
Results of the analyses will allow to compare both the clinical (patient improvement) and cost effectiveness (economic costs of the intervention) between the experimental intervention and TAU. Besides answering our primary question ('is the experimental intervention at least as effective as providing regular care, i.e. treatment as usual') these analyses are expected to provide insight into how to increase the quality and efficiency of care. The experimental intervention is expected to reduce costs and increase efficiency on (at least) the short term. The study design will also provide insight into long-term effects, possibly encouraging the implementation of an effective stepped-care model in mental healthcare.

Apart from evaluation of the effectiveness of the intervention, the study also examines what works best for the individual patient. The broad inclusion criteria allow the recruitment of patients with a wide range of mood and/or anxiety disorders. The resulting sample is then broadly representative of the patient population commonly referred to outpatient mental healthcare centers in the Netherlands. No indication of a selection bias is expected and, since the ROM method outlines patient characteristics, the advantages of the experimental intervention for the individual patient can be clearly assessed.

A major strength of this study is that it is a pragmatic randomized trial. In such trials, patients and therapist are the same as those encountered in daily practice. Care is provided by healthcare professionals from the field and, since the sample of patients is the same as those seen in daily practice, this enhances the external validity of the study. Moreover, since the stepped-care algorithm 
used covers the whole continuum from enrollment, diagnostics, assessments and treatment, this study reflects the 'real' effects of daily practice thereby allowing generalization and implementation of any beneficial logistical and organizational effects in (clinical) practice.

However, this advantage also carries some risks. Besides the logistic difficulties of implementing a pragmatic randomized trial, it is difficult to maintain treatment integrity when conducting a study in daily practice. We aim to minimize this limitation by means of our instruction meetings and supervision sessions for all clinicians and psychiatric test nurses involved. Moreover, since all treatment sessions within the brief CBT protocols are audio-taped, treatment protocol fidelity is closely examined and, hopefully, achieved.

Moreover, since conducting a study in daily practice involves the possibility of combining $\mathrm{CBT}$ and medication therapy and enhances the possibilities of non-specific effects in therapy, the potential therapeutic benefit of the intervention can only be formulated with caution.

While the design of the current study addresses many of the limitations of previous research, a preliminary reflection on further limitations and strengths of our design is required. Although homogeneous patient groups are expected in both treatment arms, the control group may have some nonspecific effects on the expected outcomes. For example, no control is made regarding the number of visits made by persons in the TAU group to their physicians. Therefore, it is possible that patients in the control group make fewer visits to their physician or, in some cases, no visits at all if they are still on a waiting list. Although this does 
reflect daily practice, we cannot rule out the nonspecific effects of an increased number of visits and/or attention from physicians as an explanation for the (possible) better outcomes in the experimental group.

Another limitation concerns the expected dropout rates at the four ROM measurement assessments, which may affect the results of the study. Although compliance with the ROM procedure is relatively successful, a $20 \%$ dropout rate is expected at reassessments. In response to these high attrition rates, the aggregated data are also analyzed according to the intention-to-treat analysis. This might yield a more valid reflection of the results and conclusions about the effectiveness of the experimental intervention.

We have described the rationale and design of an RCT examining the clinical and cost effectiveness of a time-limited, stepped-care based intervention in the treatment of mood and anxiety disorders. This study is collecting a substantial amount of data which will improve our understanding of how to develop effective strategies to adequately diagnose and treat patients with mood and/or anxiety disorders in secondary care.

If the experimental intervention proves to be as effective as regular care, this type of intervention could facilitate the growing need of providing the most optimal and (cost)-efficient mental healthcare. This study will hopefully elucidate how we can provide the most convenient and adequate mental healthcare for our patients. 


\section{Acknowledgments}

This is a collaborative study between Leiden University Medical Center and Rivierduinen (a regional mental healthcare provider) and is funded entirely by Rivierduinen. The authors thank the staff at Rivierduinen for their contribution to this research, as well as all the patients participating in this study. 


\section{References}

[1] Kessler RC, Aguilar-Gaxiola S, Alonso J, Chatterji S, Lee S, Ormel J, et al. The global burden of mental disorders: an update from the WHO World Mental Health (WMH) surveys. Epidemiol Psichiatr Soc 2009;18:23-33.

[2] Bijl RV, Ravelli A, van ZG. Prevalence of psychiatric disorder in the general population: results of The Netherlands Mental Health Survey and Incidence Study (NEMESIS). Soc Psychiatry Psychiatr Epidemiol 1998;33:587-95.

[3] Wittchen HU, Jacobi F, Rehm J, Gustavsson A, Svensson M, Jonsson B, et al. The size and burden of mental disorders and other disorders of the brain in Europe 2010. Eur Neuropsychopharmacol 2011;21:655-79.

[4] http://www.ggzrichtlijnen.nl.

[5] Buist-Bouwman MA, de GR, Vollebergh WA, Alonso J, Bruffaerts R, Ormel J. Functional disability of mental disorders and comparison with physical disorders: a study among the general population of six European countries. Acta Psychiatrica Scandinavica 2006;113:492-500.

[6] Ormel J, VonKorff M, Ustun TB, Pini S, Korten A, Oldehinkel T. Common mental disorders and disability across cultures. Results from the WHO Collaborative Study on Psychological Problems in General Health Care. JAMA 1994;272:1741-8.

[7] Bijl RV, Ravelli A. Current and residual functional disability associated with psychopathology: findings from the Netherlands Mental Health Survey and Incidence Study (NEMESIS). Psychol Med 2000;30:657-68.

[8] Mathers C, Ma Fat D, Boersma JT, World Health Organization. The global burden of disease. 2004 update. 1rd ed. Geneva, Switzerland: World Health Organization; 2008.

[9] Kessler RC, Greenberg PE, Mickelson KD, Meneades LM, Wang PS. The effects of chronic medical conditions on work loss and work cutback. J Occup Environ Med 2001;43:218-25.

[10] Murray CJ, Lopez AD. Global mortality, disability, and the contribution of risk factors: Global Burden of Disease Study. Lancet 1997;349:1436-42.

[11] Smit F, Cuijpers P, Oostenbrink J, Batelaan N, de GR, Beekman A. Costs of nine common mental disorders: implications for curative and preventive psychiatry. Journal of Mental Health Policy and Economics 2006;9:193200. 
[12] Gustavsson A, Svensson M, Jacobi F, Allgulander C, Alonso J, Beghi E, et al. Cost of disorders of the brain in Europe 2010. Eur Neuropsychopharmacol 2011;21:718-79.

[13] Hu TW. Perspectives: an international review of the national cost estimates of mental illness, 1990-2003. J Ment Health Policy Econ 2006;9:3-13.

[14] Bandelow B, Zohar J, Hollander E, Kasper S, Moller HJ. World Federation of Societies of Biological Psychiatry (WFSBP) guidelines for the pharmacological treatment of anxiety, obsessive-compulsive and posttraumatic stress disorders. World J Biol Psychiatry 2002;3:171-99.

[15] Bandelow B, Zohar J, Hollander E, Kasper S, Moller HJ, Zohar J, et al. World Federation of Societies of Biological Psychiatry (WFSBP) guidelines for the pharmacological treatment of anxiety, obsessive-compulsive and post-traumatic stress disorders - first revision. World J Biol Psychiatry 2008;9:248-312.

[16] Haaga DAF. Introduction to the special section on stepped care models in psychotherapy. Journal of Consulting and Clinical Psychology 2000;68:547-8.

[17] Davison GC. Stepped care: doing more with less? J Consult Clin Psychol 2000;68:580-5.

[18] Andrews G, Issakidis C, Sanderson K, Corry J, Lapsley H. Utilising survey data to inform public policy: comparison of the cost-effectiveness of treatment of ten mental disorders. Br J Psychiatry 2004;184:526-33.

[19] de Beurs E, den Hollander-Gijsman ME, van Rood YR, van der Wee NJ, Giltay EJ, van Noorden MS, et al. Routine outcome monitoring in the Netherlands: practical experiences with a web-based strategy for the assessment of treatment outcome in clinical practice. Clinical Psychology and Psychotherapy 2011;18:1-12.

[20] Carlier IV, Meuldijk D, van Vliet IM, van Fenema E., van der Wee NJ, Zitman FG. Routine outcome monitoring and feedback on physical or mental health status: evidence and theory. J Eval Clin Pract 2010.

[21] Meeuwissen JA, van der Feltz-Cornelis CM, van Marwijk HW, Rijnders PB, Donker MC. A stepped care programme for depression management: an uncontrolled pre-post study in primary and secondary care in The Netherlands. International Journal of Integrated Care 2008;8:e05.

[22] Bower P, Gilbody S. Stepped care in psychological therapies: access, effectiveness and efficiency. Narrative literature review. British Journal of Psychiatry 2005;186:11-7. 
[23] Shafran R, Clark DM, Fairburn CG, Arntz A, Barlow DH, Ehlers A, et al. Mind the gap: Improving the dissemination of CBT. Behav Res Ther 2009;47:902-9.

[24] van Straten A, Tiemens B, Hakkaart L, Nolen WA, Donker MC. Stepped care vs. matched care for mood and anxiety disorders: a randomized trial in routine practice. Acta Psychiatrica Scandinavica 2006;113:468-76.

[25] Van Fenema EM, van der Wee NJ, Giltay EJ, den Hollander-Gijsman ME, Zitman FG. Vitality predicts level of guideline-concordant care in routine treatment of mood, anxiety and somatoform disorders. J Eval Clin Pract 2010.

[26] van der Lem R, van der Wee NJ, van VT, Zitman FG. The generalizability of antidepressant efficacy trials to routine psychiatric out-patient practice. Psychol Med 2011;41:1353-63.

[27] American Psychiatric Association. Diagnostic and Statistical Manual for Mental Disorders- DSM-IV. 4rd ed. Washington, DC: American Psychiatric Association; 1994.

[28] Cohen J. A power primer. Psychol Bull 1992;112:155-9.

[29] de Beurs EZFG. Routine Outcome Monitoring. Het meten van therapieeffect in de klinische praktijk met webbased software. Maandblad Geestelijke Volksgezondheid 2007;62:13-28.

[30] Sheehan DV, Lecrubier Y, Sheehan KH, Amorim P, Janavs J, Weiller E, et al. The Mini-International Neuropsychiatric Interview (M.I.N.I.): the development and validation of a structured diagnostic psychiatric interview for DSM-IV and ICD-10. Journal of Clinical Psychiatry 1998;59 Suppl 20:22-33.

[31] van Vliet IM, de Beurs E. The MINI-International Neuropsychiatric Interview. A brief structured diagnostic psychiatric interview for DSM-IV en ICD-10 psychiatric disorders. Tijdschrift voor Psychiatrie 2007;49:393-7.

[32] Donker T, van SA, Marks I, Cuijpers P. A brief Web-based screening questionnaire for common mental disorders: development and validation. $\mathrm{J}$ Med Internet Res 2009;11:e19.

[33] Derogatis LR. The Brief Symptom Inventory. Baltimore, MD., Clinical Psychometric Research; 1975.

[34] Hamann J, Leucht S, Kissling W. Shared decision making in psychiatry. Acta Psychiatr Scand 2003;107:403-9. 
[35] Joosten EA, DeFuentes-Merillas L, de Weert GH, Sensky T, van der Staak $\mathrm{CP}$, de Jong $\mathrm{CA}$. Systematic review of the effects of shared decisionmaking on patient satisfaction, treatment adherence and health status. Psychother Psychosom 2008;77:219-26.

[36] Kerngroep Multidisciplinaire Richtlijnontwikkeling. Richtlijnherziening van de Multidisciplinaire richtlijn Depressie. Richtlijn voor de diagnostiek, behandeling en begeleiding van volwassen patiënten met een depressieve stoornis. Eerste revisie. Utrecht: Trimbos- Instituut; 2009.

[37] Kerngroep Multidisciplinaire Richtlijn Angststoornissen. Richtlijnherziening van de Multidisciplinaire richtlijn Angststoornissen. Richtlijn voor de diagnostiek, behandeling en begeleiding van volwassen patiënten met een angststoornis. Eerste revisie. 1rd ed. Utrecht: Trimbos- Instituut; 2009.

[38] Beck JS. Cognitive Therapy: basic and beyond. New York: Guilford Press; 1995.

[39] Shapiro F. Eye Movement Desensitization and Reprocessing (EMDR): Basic Principles, Protocols. Guilford Press; 1995.

[40] Clark DM, Salkovskis P.M. Cognitive Treatment for panic attacks: Therapist's Manual. Department of Psychiatry, University of Oxford; 1987.

[41] Butler AC, Chapman JE, Forman EM, Beck AT. The empirical status of cognitive-behavioral therapy: a review of meta-analyses. Clin Psychol Rev 2006;26:17-31.

[42] Boelens W. Behandelprotocol / Depressie / deel therapeutenboek + werkboek. 1rd ed. Amsterdam: Boom Cure \& Care; 2009.

[43] Jongh AJCMd, Broeke Et. Handboek EMDR: Een geprotocolleerde behandelmethode voor de gevolgen van psychotrauma. 1rd ed. Amsterdam: Swets \& Zeitlinger B.V.; Harcourt publishers; 2003.

[44] Koopmanschap MA, Rutten FF, van Ineveld BM, van RL. The friction cost method for measuring indirect costs of disease. $\mathrm{J}$ Health Econ 1995;14:171-89.

[45] Johannesson M, Karlsson G. The friction cost method: a comment. J Health Econ 1997;16:249-55.

[46] Smits M. Impact of policy and process design on the performance of intake and treatment processes in mental health care: a system dynamics case study. Journal of Operational Research Society 2010;61:1437-45. 
[47] van Hakkaart- Roijen LH, van Straten A, Al M, Rutten F, Donker M. Costutility of brief psychological treatment for depression and anxiety. British Journal of Psychiatry 2006;188:323-9.

[48] van 't Veer-Tazelaar $\mathrm{P}$, Smit $\mathrm{F}$, van $\mathrm{HH}$, van OP, van der Horst $\mathrm{H}$, Beekman A, et al. Cost-effectiveness of a stepped care intervention to prevent depression and anxiety in late life: randomised trial. $\mathrm{Br} \mathrm{J}$ Psychiatry 2010;196:319-25.

[49] Seekles W, van Straten A, Beekman A, van Marwijk H, Cuijpers P. Stepped care for depression and anxiety: from primary care to specialized mental health care: a randomised controlled trial testing the effectiveness of a stepped care program among primary care patients with mood or anxiety disorders. BMC Health Services Research 2009;9:90.

[50] Cape J, Whittington C, Buszewicz M, Wallace P, Underwood L. Brief psychological therapies for anxiety and depression in primary care: metaanalysis and meta-regression. BMC Med 2010;8:38.

[51] Seekles W, van SA, Beekman A, van MH, Cuijpers P. Stepped care treatment for depression and anxiety in primary care. a randomized controlled trial. Trials 2011;12:171.

[52] van 't Veer- Tazelaar $P$, van $M$, van $O$, Nijpels, van $H$, Cuijpers, et al. Prevention of anxiety and depression in the age group of 75 years and over: a randomised controlled trial testing the feasibility and effectiveness of a generic stepped care programme among elderly community residents at high risk of developing anxiety and depression versus usual care [ISRCTN26474556]. BMC Public Health 2006;6:186.

[53] de Graaf LE, Gerhards SA, Evers SM, Arntz A, Riper H, Severens JL, et al. Clinical and cost-effectiveness of computerised cognitive behavioural therapy for depression in primary care: design of a randomised trial. BMC Public Health 2008;8:224.

[54] Muntingh AD, van der Feltz-Cornelis CM, van Marwijk HW, Spinhoven P, Assendelft WJ, de Waal MW, et al. Collaborative stepped care for anxiety disorders in primary care: aims and design of a randomized controlled trial. BMC Health Services Research 2009;9:159.

[55] Jarrett RB, Rush AJ. Short-term psychotherapy of depressive disorders: current status and future directions. Psychiatry 1994;57:115-32.

[56] Ost LG. The Agoraphobia Scale: an evaluation of its reliability and validity. Behav Res Ther 1990;28:323-9.

[57] Beck AT, Steer RA. Internal consistencies of the original and revised Beck Depression Inventory. J Clin Psychol 1984;40:1365-7. 
[58] Guy W. ECDEU Assessment Manual for Psychopharmacology. National Institue of Mental Health. National Institue of Mental Health, Rockville, MD.; 1976.

[59] Bernstein DP, Stein JA, Newcomb MD, Walker E, Pogge D, Ahluvalia T, et al. Development and validation of a brief screening version of the Childhood Trauma Questionnaire. Child Abuse Negl 2003;27:169-90.

[60] Livesley WJ, Jackson DN. Manual for the dimensional assessment of personality pathology- basic questionnaire (DAPP-BQ). Port Huron: Sigma Press; 2002.

[61] Weissman AN. The Dysfunctional Attitude Scale. University of Pennsylvania, Dissertation; 1979.

[62] Endicott J, Spitzer RL, Fleiss JL, Cohen J. The global assessment scale. A procedure for measuring overall severity of psychiatric disturbance. Arch Gen Psychiatry 1976;33:766-71.

[63] Kok I, van WB. Client Appreciation in Mental Health Care: Manual of the Dutch Mental Healthcare Thermometer of Appreciation by Clients [in Dutch]. Utrecht, Trimbos-instituut/GGZ Nederland; 2003.

[64] Horowitz M, Wilner N, Alvarez W. Impact of Event Scale: a measure of subjective stress. Psychosom Med 1979;41:209-18.

[65] Weiss D, Marmar C. The impact of Event Scale-Revised. Wilson, J. and Keane, T. Assessing Psychological Trauma and PTSD. New York: Guilford.; 2009.

[66] Obsessive Compulsive Cognitions Working Group. Psychometric validation of the Obsessive Beliefs Questionnaire and the Interpretation of Intrusions Inventory: Part I. Behav Res Ther 2003;41:863-78.

[67] Liebowitz MR. Social phobia. Mod Probl Pharmacopsychiatry 1987;22:141-73.

[68] Clark LA, Watson D. Tripartite model of anxiety and depression: psychometric evidence and taxonomic implications. J Abnorm Psychol 1991;100:316-36.

[69] Young RC, Biggs JT, Ziegler VE, Meyer DA. A rating scale for mania: reliability, validity and sensitivity. Br J Psychiatry 1978;133:429-35.

[70] Sanavio E. Obsessions and compulsions: the Padua Inventory. Behav Res Ther 1988;26:169-77. 
[71] Telch MJ, Brouillard M, Telch CF, Agras WS, Taylor CB. Role of cognitive appraisal in panic-related avoidance. Behav Res Ther 1989;27:373-83.

[72] Shear MK, Brown TA, Barlow DH, Money R, Sholomskas DE, Woods SW, et al. Multicenter collaborative panic disorder severity scale. Am J Psychiatry 1997;154:1571-5.

[73] Meyer TJ, Miller ML, Metzger RL, Borkovec TD. Development and validation of the Penn State Worry Questionnaire. Behav Res Ther 1990;28:487-95.

[74] Ware JE, Snow KK, Kosinski M. SF-36 Health Survey Manual and Interpretation Guide. Boston: New England Medical Center, The Health Institute; 1993.

[75] Beidel DC, Borden JW, Turner SM, Jacob RG. The Social Phobia and Anxiety Inventory: concurrent validity with a clinic sample. Behav Res Ther 1989;27:573-6.

[76] Hakkaart-van Roijen L, van Straten A, Donker M, Tiemens B. Manual Trimbos/IMTA questionnaire for Costs associated with Psychiatric Illness (TIC-P). Rotterdam: Institute for Medical Technology Assessment, Erasmus University Rotterdam ; 2002.

[77] Asberg M, Montgomery SA, Perris C, Schalling D, Sedvall G. A comprehensive psychopathological rating scale. Acta Psychiatr Scand Suppl 1978;5-27.

[78] Tallis F. EMMA. A Questionnaire for the Measurement of Nonpathological Worry. Personality and Individual Differences 1992;13:161-8. 


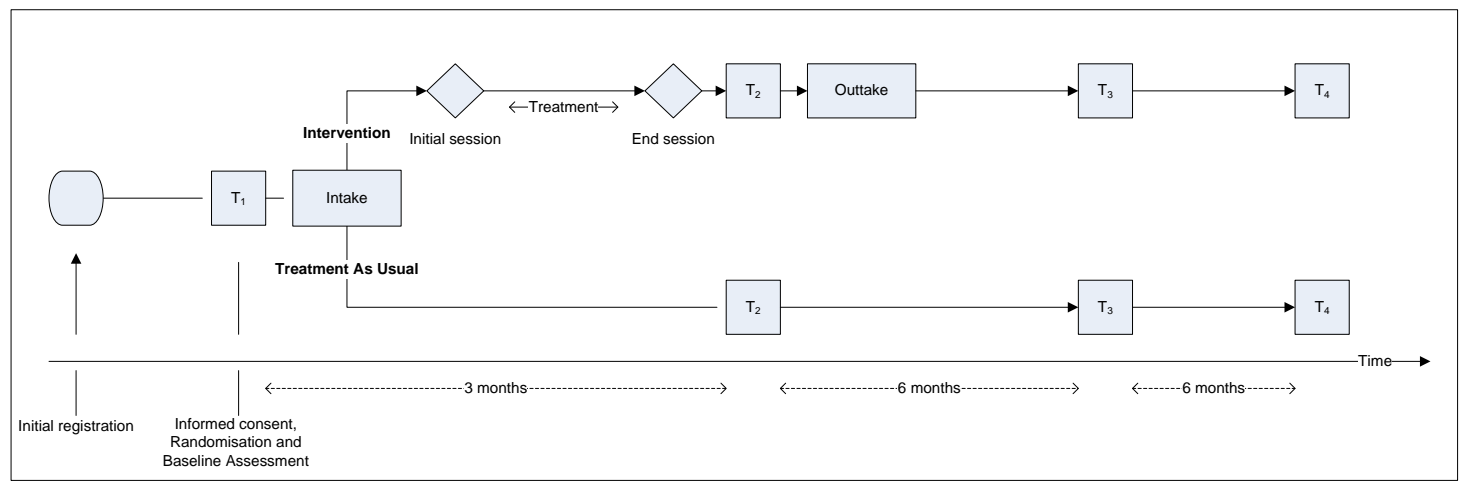

Fig. 1. Timetable of assessments during the study.

Note: $T_{1}$ : baseline assessment; $T_{2}$ : post-treatment assessment; $T_{3}$ : first follow-up assessment; $T_{4}$ : second follow-up assessment. 
Table 1. ROM study measures by time interval

\begin{tabular}{|c|c|c|c|c|c|c|c|c|c|}
\hline \multirow[t]{2}{*}{ Instrument } & \multirow[t]{2}{*}{ Full Name } & \multirow[t]{2}{*}{ Domain } & \multirow[t]{2}{*}{ Cluster } & \multirow[t]{2}{*}{$2^{a^{\text {Typ }}}$} & \multicolumn{4}{|c|}{ Time- interval ${ }^{b}$} & \multirow[t]{2}{*}{ Reference } \\
\hline & & & & & $\mathrm{T}_{1}$ & $\mathrm{~T}_{2}$ & $T_{3}$ & $\mathrm{~T}_{4}$ & \\
\hline$A G O$ & Agoraphobic Cognitions Questionnaire & Psychopath & Spec & SR & $x$ & $x$ & $x$ & $\mathrm{x}$ & {$[56]$} \\
\hline$B D I-I I$ & Beck Depression Inventory version II & Psychopath & Spec & SR & $x$ & $x$ & $x$ & $x$ & [57] \\
\hline$B S I$ & Brief Symptom Inventory & Psychopath & Gen & SR & $x$ & $x$ & $x$ & $x$ & [33] \\
\hline CGI & Clinical Global Impression & Psychopath & Gen & os & $x$ & $x$ & $x$ & $x$ & [58] \\
\hline CTQ28 & Childhood Trauma Questionnaire - 28 & Psychopath & Gen & SR & $x$ & & & & [59] \\
\hline DAPPsf & $\begin{array}{l}\text { Dimensional Assessment of Personality Pathology- } \\
\text { Short Form }\end{array}$ & Psychopath & Gen & SR & $x$ & & & & [60] \\
\hline$D A S-13$ & Dysfunctional Attitude Scale - 13 & Psychopath & Spec & SR & $x$ & $x$ & $x$ & $\mathrm{x}$ & [61] \\
\hline DEMOG & Demographic Inventory & Soc Dem & Gen & SR & $x$ & & & & \\
\hline EVALr & Evaluation Rating & Psychopath & Gen & os & $x$ & $x$ & $x$ & $x$ & \\
\hline GAF & Global Assessment of Functioning & Psychopath & Gen & os & $x$ & $x$ & $x$ & $x$ & [62] \\
\hline$H \_Q^{c}$ & Health Questionnaire & Soc Dem & Gen & SR & $x$ & & & & \\
\hline Mental Healthcare Thermometer ${ }^{d}$ & $\begin{array}{l}\text { Dutch Mental Healthcare Thermometer } \\
\text { of Appreciation by Clients }\end{array}$ & Cons Satisf & Gen & SR & & $x$ & & & [63] \\
\hline IES-R & Impact of Events Scale - Revised & Psychopath & Spec & SR & $x$ & $x$ & $\mathrm{x}$ & $\mathrm{x}$ & {$[64,65]$} \\
\hline III (Triple I) & Interpretation of Intrusions Inventory & Psychopath & Spec & SR & $x$ & $x$ & $x$ & $x$ & [66] \\
\hline LSAS & Liebowitz Social Anxiety Scale & Psychopath & Spec & os & $x$ & $x$ & $x$ & $x$ & {$[67]$} \\
\hline MASQ & Mood and Anxiety Symptom[32] Questionnaire & Psychopath & Gen & SR & $x$ & $x$ & $x$ & $x$ & [68] \\
\hline MINI-Plus 5.0.0. & Mini- International Neuropsychiatric Interview Plus 5.0.0. & Psychopath & Gen & os & $x$ & & & & [30] \\
\hline MRS & Mania Rating Scale & Psychopath & Spec & SR & $x$ & $x$ & $x$ & $x$ & [69] \\
\hline$P A D \cup A / P I-r$ & Padua Inventory revised & Psychopath & Spec & SR & $x$ & $x$ & $x$ & $\mathrm{x}$ & [70] \\
\hline PAl & Panic Appraisal Inventory & Psychopath & Spec & SR & $x$ & $x$ & $x$ & $x$ & [71] \\
\hline PDSS & Panic Disorder Severity Scale & Psychopath & Spec & os & $x$ & $x$ & $x$ & $x$ & [72] \\
\hline PSWQ & Penn State Worry Questionnaire & Psychopath & Spec & SR & $x$ & $x$ & $x$ & $x$ & [73] \\
\hline SF- 36 & Short Form Health Survey 36 & Psychosoc Func & Gen & SR & $x$ & $x$ & $x$ & $x$ & [74] \\
\hline SIAS & Social Interaction and Anxiety Scale & Psychopath & Spec & SR & $x$ & $x$ & $x$ & $x$ & [75] \\
\hline SPS & Social Phobia Scale & Psychopath & Spec & SR & $x$ & $x$ & $x$ & $x$ & {$[75]$} \\
\hline$T I C-P$ & Trimbos/iMTA questionnaire for Costs & Cons Satisf & Gen & SR & $x$ & $x$ & $x$ & $x$ & {$[76]$} \\
\hline
\end{tabular}




\section{associated with Psychiatric IIIness}

Abbreviated Comprehensive Psychopathological Rating Scale

\section{Worry Domains Questionnaire}

Web Screening Questionnaire

for common mental disorders
Cost-effect

Psychopath

Psychopath

Psychopath

$\begin{array}{lllllll}\text { Gen } & \text { OS } & X & X & X & X & {[77]} \\ \text { Spec } & \text { SR } & X & X & X & X & {[78]} \\ \text { Gen } & \text { SR } & X & & X & X & {[32]}\end{array}$

WSQ

${ }^{a} S R=S e l f$ Report; OS=Observer Scale; ${ }^{b} T_{1}$ : baseline assessment; $T_{2}$ : post treatment assessment; $T_{3}$ first follow-up asse Questionnaire H_Q (in Dutch: Gezondheidsvragenlijst); ${ }^{d}$ Mental Healthcare Thermometer (in Dutch: GGZ Thermometer)

Note: A list of all ROM measures is available at http://www.lumc.nl/psychiatry/ROM-instruments 
Table 2. Socio-demographic and clinical characteristics of the eligible patients at baseline.

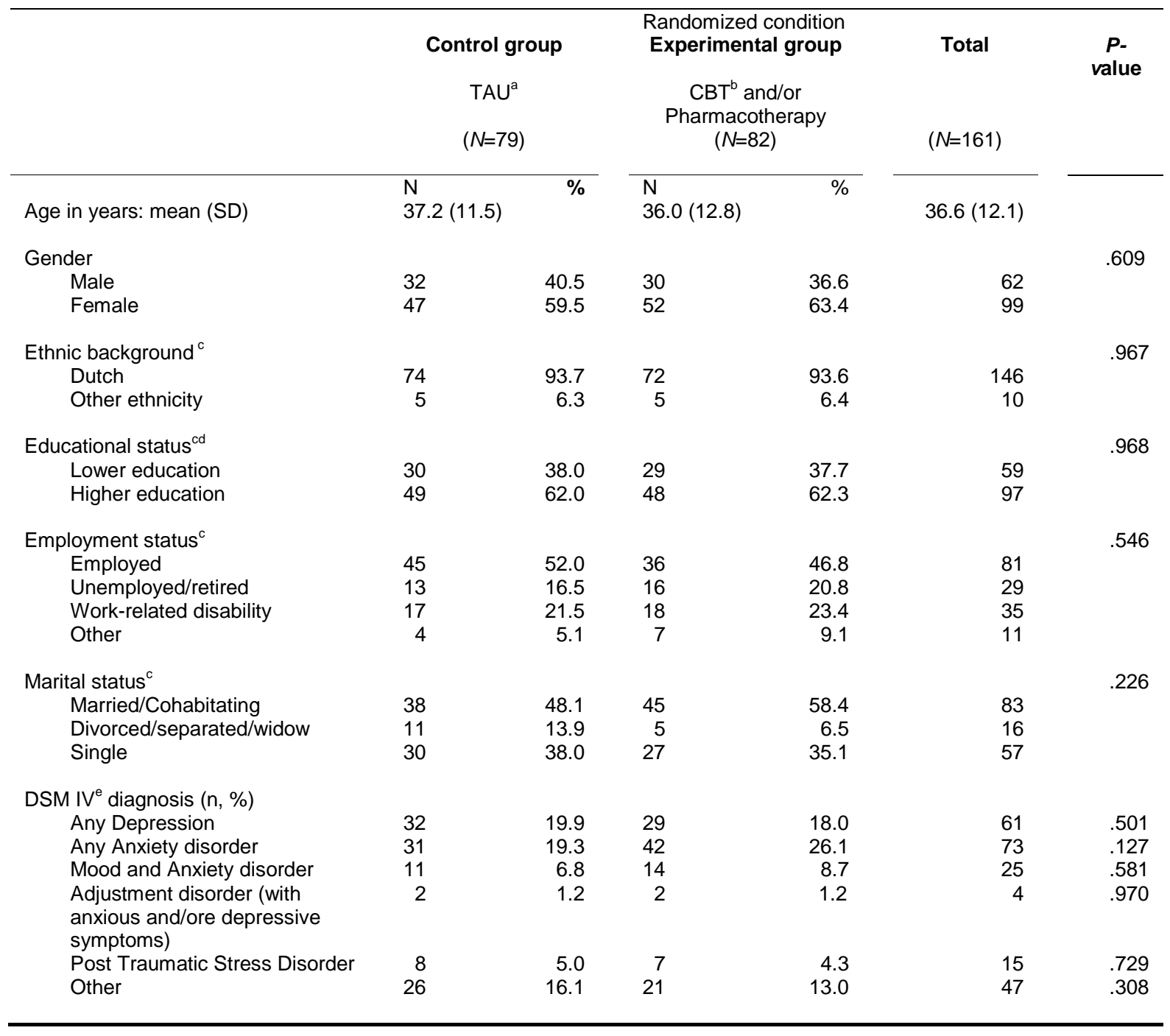

${ }^{\mathrm{a}} \mathrm{TAU}=$ Treatment As Usual; ${ }^{\mathrm{b}} \mathrm{CBT}=$ Cognitive Behavioral Therapy; ${ }^{\mathrm{c}}$ Demographic data; ethnic background, educational status and employment status are missing for 4 participants; ${ }^{d}$ Lower education= basic education, primary education, no education at all. Higher education= Higher education, university; ${ }^{e}$ DSM IV=Diagnostic Statistical Manual (of mental disorders) $4^{\text {th }}$ version.

Note: patients may have more than one diagnosis 
